\title{
LOS ESTUDIOS SOBRE EDUCACION Y SOCIEDAD EN COLOMBIA
}

Eduardo Vélez

\section{INTRODUCCION}

La rápida acumulación de saberes hace necesario realizar un inventario periódico de los resultados producidos por la investigación en un área del conocimiento de una disciplina científica. Estos inventarios denominados "Estados del Arte" son cada vez más frecuentes y populares y se están produciendo a diferentes niveles que van desde el concienzudo análisis de la naturaleza de las hipótesis y teorías producidas hasta la simple enumeración descriptiva de los resultados encontrados.

En el caso de la relación entre educación y sociedad el problema se torna exigente ya que por un lado, los resultados, debido a la deficiente estructura de difusión, están muy dispersos; por otro, porque hay un notorio desacuerdo de opiniones -tanto teóricas como metodológicas- de los trabajos existentes; y, finalmente, porque al defiinir lo social muchos son los aspectos que se deben considerar. En efecto, cuando se habla de la relación entre educación y sociedad se refiere a la educación que responda a las necesidades de bienestar de la persona, y a las necesidades de desarrollo y de progreso de las sociedades y exigencias del mundo actual. En particular, se pueden destacar las interrelaciones de la educación con la cultura, la ética, la comunicación, el mundo del trabajo, la estratificación social, la ciencia y la tecnología. Se puede llegar a niveles específicos como por ejemplo la relación entre educación y salud, fecundidad, movilidad social, la mujer, migración, nutrición, etc.

Otro aspecto que puede introducir complicaciones en una revisión, es la naturaleza de la relación educación y sociedad. Sin creer conveniente entrar en una discusión teórica, pensamos que la naturaleza del sistema educativo se determina principalmente por la naturaleza de la sociedad donde se desarrolla, pero a su vez, una educación bien concebida tiene un enorme potencial para afectar la sociedad. En efecto, a partir de la década pasada, los decisores de política ha evaluado la educación no sólo por su contribución al crecimiento (bajo el supuesto tradicional de que el desarrollo se mide con base en ingresos agregados y per cápita), sino también por su efecto en los niveles de empleo, distribución de ingresos y en la pobreza general.

Sin embargo, recientes resultados indican que las inversiones en educación deben estudiarse cuidadosamente ya que muchos países en desarrollo han experimentado una desilusión en lo referente al valor económico de la educación dado que los déficits de mano de obra de los años 1960 han sido reemplazados por un creciente desempleo entre las personas educadas, y porque a pesar de las grandes inversiones en educación aún persiste una pobreza y unas desigualdades de bienestar y oportunidades.

Por ésto, los países deben ser realistas acerca de lo que deben esperar del sistema educativo y sobre los recursos que deben invertir en educación. Estos dos aspectos son importantes pero, pueden serlo en mayor o menor grado, de acuerdo con el estado relativo de desarrollo de un país, siempre teniendo en cuenta que el sistema educativo puede incrementar o disminuir las desigualdades. No obstante, nunca se debe pensar que

\footnotetext{
* Director adjunto, Instituto SER de Investigación Ph. D en Sociología. Universidad de Illinois.
} 
la educación por sí sola va a solucionar los problemas de inequidad de una sociedad ya que estos tienen causa en muy variadas fuentes, tales como la naturaleza del mercado de trabajo, sistemas de incentivos distorsionados, abusos de poder, etc; personalmente creemos que en el pasado se ha sobre identificado a la educación (principalmente a la formal) como mecanismo de desarrollo; se ha esperado más de lo que en realidad puede proporcionar.

Colombia, de acuerdo con su ingreso per cápita y su población se ha convertido en un país de tamaño e ingresos medianos. Pero a pesar del progreso que ha tenido en los últimos veinte o treinta años, su sistema educativo aún no ha podido resolver una serie de debilidades. Entre las más agudas podrían señalarse la muy deficiente cobertura de la educación en áreas rurales y en general la calidad de la educación.

De acuerdo con información del Banco Mundial, Colombia durante la década pasada, a pesar de ser uno de los países a la cabeza de los 23 países clasificados como de ingresos medios, por estudiante de primaria solo invirtió cerca del 60 por ciento del promedio invertido por este tipo de países, y alrededor del 50 por ciento por estudiante de secundaria. En efecto, la relación entre costos unitarios de la educación y el producto Interno Bruto per cápita es bastante bajo en esos dos niveles (alrededor del 7.0 y del 14.0 por ciento respectivamente).. La diferencia también se observa cuando se compara con el promedio de los países latinoamericanos (11 y 22 por ciento para el nivel primario y el secundario respectivamente) o con el promedio de los países en desarrollo en su totalidad (15 y 52 por ciento). OSPE, 1980.

Por otro lado el gasto total en educación formal al final de los años 70 (público y privado), no alcanzó al 3.3 por ciento del PIB y parece que ha venido decreciendo; y mientras el gasto en educación pública creció a una tasa anual del 4.9 por ciento en la década pasada, el PIB lo hizo a una tasa del 4.9 por ciento anual. (DNP, 1979). Estos datos no se comparan con el promedio de lo invertido en los países en desarrollo. La mediana del gastos público en educación en 1980 en estos países era cerca del 4.0 por ciento del PIB, mientras que en Colombia en 1978 la expansión del gasto en educación era menor del 2.7 por ciento del PIB, la proporción correspondiente a los 23 países comparables era del 4.2 por ciento. Sin embargo, en Colombia el total de gastos (ordinarios y de capital) en educación son el 21.5 por ciento del gasto público total; y el total de gastos ordinarios en educación son el 27.7 por ciento del gasto público total (Unesco, 1985).

Precisamente esta situación ha hecho recapacitar a los investigadores, planificadores y decisores de políticas sobre el papel de la educación en el desarrollo social y por eso los trabajos sobre las relaciones entre educación y sociedad han venido incrementándose de manera notoria tanto en los países desarrollados como en desarrollo. En Colombia ya se ha identificado la conveniencia de estudiar los problemas educativos para encontrarles soluciones adecuadas, y el papel que las agencias internacionales principalmente el Banco Mundial, el PNUD, y la UNESCO han jugado en el desarrollo de la institucionalización de la investigación educativa, es de mucha relevancia.

Cuando se habla de investigación en educación, hasta fines de la década pasada, generalmente la temática principal se orientaba hacia las estructuras administrativas y de control obedeciendo a la necesidad de estudiar problemas relacionados con la organización y desarrollo del sistema educativo (ver Toro y Lombana, 1978). Seguía, en orden de importancia, el tema referido a la relación entre educación y sociedad generalmente orientada al estudio de la relación del sistema educativo con el medio. En 
un tercer lugar, pero con prácticamente la misma relevancia, se encontraban temas relacionados con los procesos de enseñanza -aprendizaje. Y por último se observaban temas relacionados con el contexto de la educación, los profesores y los alumnos.

Con base en los Resumenes Analíticos en Educación, RAE, (números 1 al 5) se puede completar una visión de la distribución de los temas de las principales investigaciones educativas a partir de 1978 y hasta 1986. Esta parece no haber cambiado sustancialmente, ya que los temas sobre administración y control siguen teniendo una ventaja, aunque tiende a cerrarse. En efecto, ya los temas sobre educación y sociedad parecen aventajar a los relacionados con el proceso enseñanza-aprendizaje. En la cuarta posición aparecen las investigaciones de tipo evaluativo.

Otro aspecto importante es que la tendencia observada parece indicar que el enfoque de la investigación en educación en Colombia ha pasado de la descripción de aspectos macros y de la estructura de la planeación educativa a la consideración de aspectos relacionados con la calidad y con fenómenos relacionados con la estructura educativa, tales como el trabajo, la migración, la participación política, etc.

En esta revisión nos ocuparemos de los temas clasificados como de educación y sociedad, algunos clasificados como evaluativos pero que conciernen a la sociología de la educación, lo mismo que algunos que atañen a problemas de calidad, fenómenos específicos tales como el trabajo, la migración, etc.

Vale la pena anotar que a pesar del mejoramiento de la calidad de la investigación realizada y del uso cada vez más frecuente de metodologías más adecuadas, se siguen observando deficiencias tanto en lo referente a la formulación de los problemas a investigar como en el uso de los diseños metodológicos y en los niveles de análisis. En realidad hay un nivel heterogéneo en cuanto a este aspecto se refiere y así como existen trabajos de muy dudosa calidad, hay otros que satisfacen los más exigentes requisitos académicos.

Aunque hay varías posibilidades de clasificar los diferentes trabajos sobre educación y sociedad, no creemos que hay una mejor que otras; todo depende del propósito de la clasificación. Generalmente se puede clasificar dependiendo de los marcos teóricos que les dan consistencia conceptual; --funcionalismo, estructuralismo, marxismo, etc.--; también se clasifican por disciplinas académicas - sociología, antropología, historia, arte-; otros lo hacen por la técnica de investigación utilizada --investigación documental, investigación-acción, encuestas por muestras, etc.-; y finalmente se puede clasificar por áreas o tópicos de investigación dentro de una o varias disciplinas académicas

--educación y movilidad social, educación y comportamiento político, calidad del docente y logro cognoscitivo, etc.

Se debe observar que una contabilización de los trabajos que pueden considerarse como estudios socioeducativos realizados en Colombia en un período de 75 años (hasta 1980) --ver Facundo (1980)--, ha identificado más de 910 títulos. El autor aclara que la selección pretende abarcar el universo de los trabajos realizados independiente de su calidad, extensión, profundidad, de si se han publicado o no y de su tratamiento metodológico. Al clasificarlos según su objeto de análisis encuentra que la mayoría de ellos, el 80.3 por ciento, se concentra en análisis globales o parciales del sistema educativo dentro de la orientación de la sociología de la educación que el autor señala como aquella cuyo objeto central es el sistema educativo como una organización social específica, sus funciones sociales, estructuras y condiciones. Otros trabajos se enmarcan 
dentro de la orientación de la sociología de la educación cuyo objeto central es la educación como función profesional; diferentes grupos sociales (maestros, alumnos) con el 10.5 por ciento de los trabajos; la educación como función del proceso de reproducción general de la sociedad, que abarca el 5.8 por ciento de los trabajos; y la educación como función colateral de las diferentes instituciones sociales, que cubre el 3.4. Por ciento de los trabajos.

Esta perspectiva de clasificación es otra alternativa que podría utilizarse en el contexto de una revisión de los trabajos sobre la relación educación y sociedad, pero la descartamos en nuestro caso debido a que siendo nuestro interés enmarcar los estudios existentes dentro del contexto de la rentabilidad de la educación, hay más claridad y utilidad en la exposición si la clasificación permite una mejor identificación de los productos de la educación

Para los propósitos de nuestro trabajo no tiene sentido clasificar los estudios por sus marcos teóricos generales (Inclusive porque hay notorios vacíos con respecto a algunos de ellos) o por teorías más específicas que son de interés a la investigación, tales como la teoría del capital humano o la teoría de la estratificación social. Tampoco creemos oportuno hacer una clasificación por técnicas de investigación utilizada. Por exclusión hacernos uso de una clasificación que combine las disciplinas académicas con temas específicos; aunque somos conscientes de las limitaciones y dificultades que se pueden presentar ya que muchos trabajos pueden referirse de manera simultánea a varios temas o disciplinas.

\section{EDUCACION Y CULTURA}

El primer aspecto a considerar se refiere a la relación entre educación y cultura. Curiosamente aunque el concepto de cultura es muy utilizado en el contexto de los medios masivos de comunicación, está lleno de grandes ambigüedades, en parte porque ha sido estudiado por diversas disciplinas científicas tales como la antropología, la historia, la filosofía, la sociología, y cada una de ellas le da una connotación particular. Desde el punto de vista sociológico podemos citar a Mannhein (1957) quien define la sociología de la cultura como el estudio de las funciones mentales en el contexto de la acción y que tiene como objeto de análisis al estudio de los procesos mentales en el contexto social. Se sitúa esta forma de pensamiento en la tradición Weberiana la cual argumenta que la acción social se encuentra básicamente en el significado que los actores da, individual o colectivamente, a sus acciones. Alvarado (1980) cita al sociólogo francés Paul Chombart de Lauve quien reconoce que la cultura es un conjunto complejo de representaciones, creencias y valores en constante transformación en la vida cotidiana que tiene sus génesis en los cambios tecnológicos y económicos que operan en una sociedad y a su vez se constituye en el motor principal del cambio social. Esta posición complementa la anterior y aclara el concepto de la importancia de las estructuras mentales y su relación con la acción. Para una aplicación empírica de esta posición ver Inkeles y Smith (1974).

La posición sociológica se complementa con la posición pedagógica, la que considera a la educación como el factor más importante que la cultura tienen para el desarrollo de la persona en la sociedad. Alvarado (1980, pp 12-13) integrando estas posiciones y nuevamente con base en Chombart de Lauve señala que" La educación como institución social, juega un papel fundamental dinamizando o frenando la cultura, convirtiéndose así en arma de doble filo: imponiendo los modelos dominantes; manipulando las aspiraciones, 
asegurando la reproducción de las estructuras y el mantenimiento de los valores tradicionales como instrumento de los grupos dominantes en una sociedad; o bien, siguiendo el proceso inverso a partir de los propios sujetos -actores y a través de un proceso de auto-educación, logrando una participación crítica pero activa en la estructura social, política y económica."

En Colombia, salvo algunas monografías de grado, principalmente de la Universidad Pedagógica Nacional, existen pocos estudios sobre la relación educación cultural desde el punto de vista puramente pedagógico; prácticamente no existen estudios sobre esta relación desde el enfoque antropológico; y solo unos pocos desde la perspectiva histórica. La gran mayoría de los estudios sobre la relación educación -cultura son de corte sociológico o psicosociológico.

Los estudios históricos tratan de señalar el sistema de valores a través de la evolución del sistema educativo. Cronológicamente un primer trabajo que relaciona los dos conceptos es el de Porras Troconis (1952) cuando escribe una reseña histórica acerca de lo que fue el sistema educativo durante la Nueva Granada. Sobre el mismo tema trabaja Nieto (1955), quien propone algunos elementos de tipo filosófico sobre el sistema educativo durante la Colonia.

Fals Borda (1962), en un trabajo histórico-sociológico, estudia la naturaleza y las tendencias de algunos valores educativos importantes para entender situaciones concretas del país desde la Colonia hasta la década de los 50.

Bernal et al (1965) también desde un contexto histórico-sociológico analizan la evolución de la educación y la cultura en Colombia a partir del periodo pre-colombino hasta el principio de la década de los setentas. En un capítulo tratan el tema concreto de la relación educación-cultura analizando, con cierto detalle, el sistema de valores predominantes que inspiraba la naturaleza de la educación.

Más recientemente Silva (1981) en un detallado estudio sobre las reformas educativas entre 1767 y 1790 analiza la relación entre algunas posiciones filosóficas y los planes de estudio de la época y las barreras que las estructuras sociales imponían a ciertas reformas educativas.

El mismo autor, Silva (1985) dentro de la perspectiva de la historia social de la cultura, estudia procesos de selección social de los estudiantes y profesores universitarios y explora aspectos referentes al destino social de los primeros.

A pesar de la existencia de trabajos que estudian la relación sin embargo, anotamos que en el país no parece existir una tradición al respecto, y fuera de las monografías de grado señaladas, solo son esporádicos los trabajos sobre el tema. Vale la pena mencionar el de Muñoz (1983) donde se analiza el aprendizaje como una de las formas de partir de la cual se logra la apropiación de una determinada cultura y se postula el concepto de des-socialización infantil para aludir a una deficiencia en el proceso de endoculturación y plantear la necesidad de actuar con el objeto de mejorarla. Igualmente se puede citar al mismo autor, (Muñoz, 1984) donde plantea el desarraigo cultural de la escuela en nuestro medio y concluye que los estudios están fuera del contexto de la realidad cultural originando, en parte el fracaso escolar.

Otro trabajo que amerita reseñarse es el de Martínez (1984) donde se enfatiza el hecho de que la escuela (como institución) en la Nueva Granada surge como un lugar 
aislado de la familia y con un saber propio referente a la enseñanza, donde se transmiten saberes sobre el comportamiento del hombre para con Dios y para con la sociedad. También resalta la formación, desde esa época, del saber pedagógico en lo referente a reglas y métodos para enseñar y sugiere la formación de otra escuela y otra manera de ser maestro a los que la pedagogía les confiere identidad cultural.

Gómez et al (1985) en un trabajo que también se podría clasificar como sociopsicológico, analizan la importancia de las actitudes en los procesos de cambio en las instituciones educativas y muestran que entre los docentes hay una preferencia por la autoridad, lo cual se explica por el papel ideologizante que ha producido la escuela.

Por último, un estudio hecho por Flórez y Batista (1982) se concentra en las concepciones pedagógicas que orientan la práctica pedagógica de los maestros de educación primaria oficial y concluye que el pensamiento del magisterio es tradicionalista que recoge el prejuicio aristocrático contra las "artes útiles", su meta pedagógica es el aprendizaje de los conocimientos generales, valores y habilidades más o menos estéticas, heredadas del pasado clásico y humanista de occidente. Esto se logra por medio de un método academicista, verbalista y escolástico dentro de un medio disciplinario.

La gran mayoría de los estudios sobre educación y cultura se han desarrollado desde el punto de vista sociológico. Cataño (1974 y 1980) señala que Wiliamson (1962), con su estudio sobre la descripción del estudiante universitario y su ideología, fue el punto de partida para este tipo de trabajos. En verdad tuvo un efecto de demostración reflejado en algunas monografías de grado en el Departamento de Sociología de la Universidad Nacional entre los cuales señalamos la de Rodríguez (1967) quien estudia la naturaleza de la relación entre la educación secundaria y las actitudes hacia el cambio social, encontrando que existe una relación inversa entre nivel socioeconómico y actitudes hacia el cambio. Mas tarde el mismo autor Rodríguez (1970), estudia papel que juega la Iglesia en la socialización y en el campo educacional.

En la misma época, Benoit (1968) adelanta un estudio para analizar las funciones ideológicas del bachillerato por medio de las actitudes de los estudiantes de último año sobre la secularización, la democratización y la emancipación.

Alvarado (1973) analiza las aspiraciones culturales de la clase obrera en Bogotá, y encuentra que en dicho estrato la imagen del hombre culto se identifica con la del hombre instruido. Esta imagen a su vez se condiciona por la cultural dominante, y por lo tanto concluye que las aspiraciones culturales de los obreros son producto impuesto por la cultura de la clase dominante.

En un estudio de corte psico-sociológico sobre la motivación en el individuo, Sudarsky (1974) establece que algunas variables sociológicas entre las que se encuentra la educación, determinan las motivaciones sociales (aspectos de la cultura).

Merecen particular atención los estudios que se han hecho sobre la Acción Cultura Popular, ACPO* en particular en lo relacionado con adquisición de valores modernos. Bernal (1976) por medio de caso mide cualitativamente el impacto de esta forma de educación no formal en las estructuras mentales del campesino, encontrando, entre otras cosas, que la capacidad de liderazgo se asocia con el proceso educativo.

El mismo autor (ver Bernal 1978) considera el proceso educativo de ACPO como uno por medio del cual se desarrolla el individuo conmo persona y como parte de la sociedad 
y se basa en el supuesto de que la educación establece la base fundamental a partir de la cual la persona debe continuar su aprendizaje adaptándose a las condiciones concretas de su medio ambiente.

\section{ECONOMIA Y EDUCACION}

Un segundo tema a considerar se refiere a la relación entre economía y educación. Concretamente incluiremos algunos hallazgos de los principales estudios sobre los mercados de trabajo, la distribución del ingreso, la eficiencia externa, la formación en relación con la demanda, y la rentabilidad económica de la educación.

Tópico central a este tema general es el referente a la relación entre la educación y los ingresos. Cuando se analizan funciones de ingresos, ya sea en países desarrollados o en desarrollo, se ha encontrado sistemáticamente una correlación positiva entre años de educación formal e ingresos y una correlación positiva entre años de experiencia e ingresos. Psacharopoulos (1973; 1981) por medio de tasas de rendimiento ha mostrado la asociación positiva entre educación e ingresos. Colombia es uno de los pocos países en desarrollo que presenta la oportunidad de mirar funciones básicas de ingresos de forma longitudinal para los últimos veinte años. En el cuadro 1 se presentan varias funciones básicas de ingreso desde 1965 hasta 1984. Los signos de los coeficientes confirman lo estipulado por la teoría del capital humano; y a pesar de que las muestras comparadas no son de los mismos universos, se confirma la hipótesis de que invertir en capital humano representa un rendimiento decreciente en el tiempo. Con la expansión experimentada en la educación durante los últimos veinte años se observa un menor impacto de la educación sobre los ingresos.

Mientras que para los empleados en 1965 (solo para Bogotá) la tasa de rendimiento de la educación era de 17.3 por ciento, y en 1974 (solo para Bogotá) era de 12.8 por ciento, en 1984 (para ciudades más importantes incluyendo Bogotá) llegaba a ser del 11.0 por ciento. Para el caso de los empleados en el sector privado, la tasa de rendimiento de la educación, baja de 14.6 por ciento en 1974 a 10.6 por ciento en 1984; y para los empleados del sector público el descenso es de 3.9 puntos en el mismo periodo, bajando de 13.4 al 9.5 por ciento. Sin embargo esta disminución de los rendimientos son mas notorios entre los asalariados, ya que para los cuenta propia (independientes) el rendimiento de un año de educación adicional permanece bastante similar en los últimos diez años; pasa del 13.2 por ciento en ¡974 al 12.9 por ciento en 1984 , una caida de solo 0.3 puntos.

El control que los últimos gobiernos han tenido que poner a los incrementos salariales, en especial a los empleados del sector público, como parte de políticas antiinflacionarias, puede ser un factor explicativo de que haya sido precisamente a nivel de empleados entre quienes más se haya notado la disminución de la tasa de rendimiento de la educación.

En lo referente a la educación no formal, se ha realizado algunas evaluaciones del SENA que han producido resultados igualmente conformatorios de la teoría del capital humano. (VER Maldonado 1973; Puryear, 1977; y Jiménez y Kugler 1986). El trabajo de Jiménez y Kugler, el más comprensivo al respecto, utilizando una muestra de 1410 egresados de varios tipos de cursos y de una muestra de 1023 casos de control definida como miembros ocupados en la fuerza laboral con oficios similares dentro de empresas similares a los correspondientes a los egresados del SENA, demuestra, por medio de una función de ingresos expandida (incluye características socioeconómicas de los padres, 
una variable dummy de pertenencia o no al SENA), que la tasa de redimiendo de la educación es cercana al 11 por ciento y cl haber estudiado en el SENA implica una ganancia de mas del 13 por ciento en los ingresos en comparación con sus similares.

Otro trabajo relativamente reciente (ver Psacharopoulos, Vélez y Zalbaza, 1986) compara las tasas de rendimiento social del bachillerato contrastando las de la educacion media diversificada (INEM) con las de la educación univocacional. Los egresados INEM tienen una tasa de rendimiento sobre la educación primaria de 9.5 por ciento frente a una de 9.7 por ciento para los egresados de escuelas univocacionales, sugiriendo que son básicamente iguales.

Un análisis de los determinantes de los ingresos de una muestra de asalariados del sector manufacturero en Colombia (ver Caillodsy Briones, 1981), concluye que el número de años de estudio es la variable que mejor explica la varianza de los salarios. Mientras que un modelo socioeconómico explica el 60.5 por ciento de la varianza, la educación sola explica el 28.7 por ciento. Otros factores que intervienen son, en su orden, la edad, la capacitación profesional, el sexo, y, por último, la experiencia dentro de la empresa. Al incluir en el modelo algunas características de la empresa, la varianza explicada llega a ser el 67.2 por ciento y naturalmente se disminuye el impacto de la educación; sin embargo, sigue siendo la principal variable explicativa. Resalta, como un importante factor explicativo del ingreso, el número de personas bajo supervisión.

Mohan (1987) analizando datos de trabajadores para la ciudad de Bogotá encuentra que la educación es, en forma amplia, la característica que mejor explica la distribución de los ingresos, inclusive mucho más que la experiencia.

Este resultado corrobora la importancia de la educación en la explicación de los ingresos de los asalariados urbanos. En adición a los trabajos citados vale la pena señalar el análisis de Kugler (1980) que utilizando una submuestra de la Encuesta Nacional de Hogares - Etapa 5 de 1971, y un modelo socioeconómico, concluye que la educación es el mejor determinante de los ingresos, más que la experiencia laboral, el ingreso o la ocupación y educación de los padres el sexo el origen rural, etc. Señala el autor la importancia de aislar el efecto neto de la educación del asalariado porque esta a su vez es explicada por las características socioeconómicas de su familia. Este análisis, que no se ha realizado, podría hacerse utilizando la técnica del análisis de caminos, de frecuente uso en la literatura sociológica americana y cada vez más próximo a las necesidades del análisis económico.

Uno de los pocos trabajos sobre la rentabilidad de la educación rural en Colombia es el de Haller (1972) quien basándose en la producción agropecuaria concluía que no había un efecto directo de la escolaridad sobre los ingresos; solo al considerar la utilización de tecnología moderna se encontraba una relación importante ya que en Espinal, Tolima, la tasa de rendimiento social para los cinco años de educación primaria llegaba a ser cercana al 60 por ciento.

Más recientemente con una muestra de 2396 hogares rurales, Bonilla y Vélez (1987), encuentran en un modelo de regresión múltiple que el nivel educativo del jefe del hogar es la Variable que mejor explica los niveles de su ingreso.

Igualmente, con excepción de la estructura de edades del hogar, es la mejor variable para explicar el ingreso familiar total. 
Un análisis más detallado de esta misma muestra, (ver Aguilar y Perfetti, 1987), basado en regresiones de capital humano encontró que la educación es una fuente de generación de ingresos, en particular para los hogares que obtienen rentas salariales. Igualmente, para los hogares que reciben ingresos por actividad agropecuaria se comprueba la hipótesis del capital humano ya que tanto la educación como la experiencia del jefe de hogar tienen una influencia positiva y significativa en la generación de ingresos.

Este hallazgo se complementa con lo encontrado por Vélez et al (1986) con una muestra de hogares rurales productores de café en el sentido que los ingresos por venta de café se explican en un modelo socioeconómico por variables estructurales (región), por el tamaño de la explotación, por el nivel educativo del hogar (coeficiente estándar de regresión es 0.11 ) y otras características.

En Colombia, es resumen, los trabajos realizados sobre la rentabilidad de la educación (o la relación educación-salarios) son similares a los encontrados en otros países en desarrollo. Por un lado, en cuanto a las magnitudes, y, por otro, en cuanto a los niveles. Selowsky (1969), por ejemplo encontró una mayor rentabilidad para la educación primaria (28 por ciento) que para la secundaria (20 por ciento) y que para la universitaria (6 por ciento). Resultados similares se encuentran en Dougherty (1971), que corroboran los resultados alcanzados anteriormente por Franco en $1964 .$.

Otra similitud con los hallazgos descritos en la literatura internacional, documentada en el cuadro 1 , se refiere a la tendencia decreciente de la rentabilidad de la educación en el tiempo.

Por último, a pesar de la poca información disponible, parece ser que la educación primaria rural tiene una menor tasa de rentabilidad que la educación primaria urbana. Datos del DANE citados por el Thirsk (1974). 


\begin{tabular}{|c|c|c|c|c|c|c|c|c|c|c|c|}
\hline \multirow[t]{3}{*}{$\begin{array}{c}\text { Variables } \\
\text { Independientes }\end{array}$} & \multirow{3}{*}{$\begin{array}{l}\text { Schultz } \\
\\
\text { Bogotá, } 1965 \\
\text { F. de T. }\end{array}$} & \multicolumn{3}{|c|}{ Bourgouignon } & \multirow{2}{*}{\multicolumn{2}{|c|}{$\begin{array}{c}\text { Psacharopoulos } \\
\text { Colomb. } 1975\end{array}$}} & \multirow{2}{*}{\multicolumn{2}{|c|}{$\begin{array}{c}\text { Horn } \\
\text { Colombia } 1979 \text { asalariados }\end{array}$}} & \multirow{2}{*}{\multicolumn{3}{|c|}{$\begin{array}{c}\text { Psacharopoulos } \\
\text { Arraigada y Vélez } \\
\begin{array}{c}\text { Colombia } 1984 \\
\text { Asalariado }\end{array}\end{array}$}} \\
\hline & & \multirow{2}{*}{$\begin{array}{l}\text { Bogotá, } \\
1971 \\
\text { Fuente de } \\
\text { Trabajo }\end{array}$} & \multicolumn{2}{|c|}{ Bogotá, 1974} & & & & & & & \\
\hline & & & Asalariado & $\begin{array}{l}\text { Cuenta } \\
\text { propia }\end{array}$ & S. Públicos & S. Priv & Púb.Priv & Cta prop. & Públic. & Priv. & $\begin{array}{l}\text { Cuenta } \\
\text { propia }\end{array}$ \\
\hline $\begin{array}{c}\text { Años de } \\
\text { educación }\end{array}$ & $\begin{array}{l}0.173^{*} \\
(13.4)\end{array}$ & $\begin{array}{l}0.167^{*} \\
(38.9)\end{array}$ & $\begin{array}{l}0.127^{*} \\
(31.1)\end{array}$ & $\begin{array}{l}0.132^{*} \\
(12.0)\end{array}$ & $\begin{array}{l}0.134^{*} \\
(44.2)\end{array}$ & $\begin{array}{l}0.146^{*} \\
(73.8)\end{array}$ & $\begin{array}{l}0.129 * \\
(30.5)\end{array}$ & $\begin{array}{l}0.126^{*} \\
(55.3)\end{array}$ & $\begin{array}{l}0.95^{*} \\
(40.7)\end{array}$ & $\begin{array}{l}0.106^{*} \\
(81.8)\end{array}$ & $\begin{array}{l}0.129^{*} \\
(50.6)\end{array}$ \\
\hline $\begin{array}{l}\text { Experiencia } \\
\text { Laboral }\end{array}$ & $\begin{array}{c}0.121^{*} \\
(8.8)\end{array}$ & $\begin{array}{l}0.078^{*} \\
(17.6)\end{array}$ & $\begin{array}{l}0.67^{*} \\
(22.5)\end{array}$ & $\begin{array}{c}0.057^{*} \\
(8.1)\end{array}$ & $\begin{array}{l}0.041 \\
(11.2)\end{array}$ & $\begin{array}{l}0.070 * \\
(32.3)\end{array}$ & $\begin{array}{l}0.055^{\star} \\
(10.4)\end{array}$ & $\begin{array}{l}0.068^{*} \\
(27.5)\end{array}$ & $\begin{array}{l}0.034^{*} \\
(15.9)\end{array}$ & $\begin{array}{c}0.058 *(4 \\
3.6)\end{array}$ & $\begin{array}{l}0.057^{*} \\
(19.3)\end{array}$ \\
\hline $\begin{array}{l}\text { Exp. Al } \\
\text { cuadrado }\end{array}$ & $\begin{array}{l}-.001^{*} \\
(7.3)\end{array}$ & $\begin{array}{c}-0.001^{*} \\
(12.6)\end{array}$ & $\begin{array}{l}-.000^{*} \\
(15.8)\end{array}$ & $\begin{array}{c}-.000^{*} \\
(7.3)\end{array}$ & $\begin{array}{c}-.000^{*} \\
(7.3)\end{array}$ & $\begin{array}{l}-.001^{*} \\
(23.3)\end{array}$ & $-.001^{*}(7.0)$ & $\begin{array}{l}-.001^{*} \\
(19.8)\end{array}$ & $\begin{array}{c}-.0005^{\star} \\
(10.5)\end{array}$ & $\begin{array}{l}-.0007^{*} \\
(29.6)\end{array}$ & $\begin{array}{c}-.0007^{*} \\
(16.1)\end{array}$ \\
\hline Constante & 4.30 & 5.08 & - & - & 6.38 & 5.88 & 6.99 & 6.87 & 8.64 & 8.23 & 7.98 \\
\hline $\mathrm{R} 2$ & 0.88 & 0.63 & 0.39 & 0.24 & 0.61 & 0.52 & 0.54 & 0.45 & 0.43 & 0.38 & 0.29 \\
\hline $\begin{array}{c}\text { Tamaño de la } \\
\text { muestra }\end{array}$ & 722 & 1016 & 2160 & 762 & 1376 & 5507 & 816 & 3718 & 10118 & 12166 & 6676 \\
\hline
\end{tabular}

Notas: $\quad$ Valores t en paréntesis

- $\quad$ Significancia estadística, se refiere al nivel del 1 por ciento o mejor

Fuente Schultz (1968), se refiere a una muestra de la fuerza de trabajo masculina; Bourgouignon (1980) se refiere a una muestra de la fuerza de trabajo masculina y en los cuenta propia excluye a las personas con educación universitaria; Psacharopoulos (1983) se refiere a una muestra de trabajadores hombres urbanos y el sector privado incluye asalariados y cuenta propia; Horn (1986) se refiere a una muestra de trabajadores hombres urbanos; Psacharopoulos Arraigada y Vélez (1987) se refiere a una muestra de asalariados y cuenta propia masculinos urbanos. 


\section{MAESTROS Y EDUCACION.}

Otro tema de interés para esta revisión sobre la relación entre educación y sociedad se refiere a los estudios sociales de uno de los pilares del sistema educativo, los maestros. Ya Parra (1980) ha señalado la importancia de este tema por los efectos que produce el rol de los maestros en todo el sistema educativo; y sin embargo, como lo anota el mismo autor, hasta fines de la década pasada poca era la atención que se había dado al tema.

Un primer tipo de trabajos, que se puede identificar en la literatura sobre maestros, es el que se refiere a la relación entre estos y el desarrollo, sea que se consideren como obstáculos o inducidores al cambio.

Velandia et al (1975) en el contexto de la educación no formal, analiza la relación existente entre el relativo nivel de desarrollo socioeconómico y cultural de las regiones y la naturaleza de los maestros y las técnicas educativas. Parra (1979 y 1985) analizando características del maestro en diferentes contextos socioeconómicos también aporta a este tipo de estudios y muestra que existe una relación entre el desarrollo nacional y el desgaste de la imagen y del prestigio del maestro.

Otro tipo de estudios analiza las condiciones de trabajo en términos regionales (macro) y comunitarios y los efectos que tienen en el desempeño del maestro y su interacción con los alumnos.

El trabajo de Pass (1979) muestra el potencial del maestro como agente de cambio en las comunidades; otros como el de Ferro (1978) señalan la adaptación del maestro y la docencia a necesidades concretas en diferentes contextos socioeconómicos. Parra et al (1981) en un trabajo etnográfico más sistemático analizan las relaciones sociales al interior de escuelas normales en contextos sociales diferentes (urbano-rural); y más relacionado con el tema de los estudios sobre la relación comunidad-maestro, Parra y Zubieta (1981) destacan el impacto del rol del maestro y de la misma escuela en la comunidad. También con una perspectiva etnográfica, de Tezanos (1983) analiza, entre otras, las relaciones maestro-alumno y escuela-comunidad. Los resultados muestran que con respecto a la primera relación, las formas memorísticas y mecanicistas del trabajo del maestro limitan la creatividad de los niños y la interacción se basa en relaciones de obediencia. En cuanto a la segunda relación, aunque hay interacción, esta no es clara porque ni los maestros ni los padres de familia tienen claridad sobre la naturaleza del trabajo comunitario. Sin embargo, la relación escuela -comunidad se materializa en acciones concretas de beneficio mutuo. Las interacciones y sus efectos son diferentes dependiendo de la localización urbana-rural de la escuela.

Un estudio enmarcado en las actitudes políticas de los maestros --tema poco estudiado--, que analiza el rol político del docente colombiano en el aula y en la comunidad --ver Losada (1985)-- concluye que los maestros se sienten ineficaces frente al mundo político que los rodea y transmiten a los alumnos una imagen negativa del sistema político imperante. Sin embargo, desvirtúa la creencia de la militancia política de los maestros ya que estos delegan en los gremios su representación frente al Estado.

\section{FORMACION DE DOCENTES}

Un tema específico sobre la relación maestro y educación es el referente a la formación de docentes. En la literatura colombiana son frecuentes los trabajos sobre el tema pero 
generalmente son análisis parciales de algunos aspectos particulares o se orientan a grupos muy limitados.

Queremos iniciar la reseña de trabajos sobre este tema con el de Martínez (1981) sobre la historia de la aparición del oficio de maestro como reacción de la sociedad para suplir el vacío causado por la expulsión de los Jesuitas en 1767 cuando surge el oficio de maestro no ya como transmisor del alfabeto sino como iniciador de la escuela pública en el país.

Este oficio se institucionaliza como práctica pedagógica a partir de 1821 cuando se le concede un gran valor a la formación de ciudadanos; esta posición se refuerza en 1844 con la puesta en práctica del énfasis en la formación de preceptores y con la creación de escuelas normales (ver Zuluaga, 1982) proceso que cobra una fuerza mayor en la década de 1870, cuando se organizan las escuelas normales con la asesoría de pedagogos alemanes, y se consolida con la creación oficial de la primera institución de nivel universitario dedicada a la formación de profesores, la Facultad de Pedagogía de Tunja en $1933^{*}$ (ver Ocampo, 1978) y con la creación de la Facultad de Educación de la Universidad Nacional en Bogotá en 1935

Esta primera etapa del desarrollo de las Facultades de Educación (va hasta 1953) sigue un modelo educativo europeo con programas de formación de docentes de enseñanza media, organizados y controlados por el gobierno de una manera directa (DNP - MEN, 1973).

Con la fundación en 1953 de la Facultad de Educación de la Universidad de Antioquia se inicia una segunda etapa con la proliferación de facultades de educación, ya no como instituciones autónomas, sino como parte de universidades de acuerdo con el modelo norteamericano. El crecimiento fue tan notorio que el número de facultades pasó de 8 en 1960 a 16 en 1966, 48 en 1976 y 62 en 1981 (ver Eder de Zambrano et al 1982), con un correspondiente incremento en la matrícula pasando de 3862 en 1964 a 43.617 en 1977y a 44.479 en 1980.

Este crecimiento desafortunadamente fue desordenado y no garantizaba unas normas mínimas de calidad, lo cual se reflejaba en una situación crítica desde el punto de vista académico y administrativo.

La tercera etapa del desarrollo de las facultades de educación que se podría situar a partir de los años 1970, se caracteriza por ser una fase de reflexión y autocrítica que se mantiene hasta nuestros días. Se ha intentado establecer unos lineamientos para tratar de integrar los componentes pedagógicos y el conocimiento profesional, de mejorar los currículos adecuándolos --a veces con poco éxito-- a las necesidades reales del pais y. haciéndolos, por lo tanto, más pertinentes. Sin embargo, mucho es lo que se tiene que hacer para lograr esos niveles. Igualmente grave es la situación relacionada con el problema del desempleo de los licenciados en educación. Según el Ministerio de Educación Nacional (citado por Eder de Zambrano et al. 1982) en 1981 hubo más de 6300 egresados y el sistema solo absorbió 105 licenciados.

Otros problemas que se han señalado en forma reiterada son la menor calificación del docente rural, en comparación con el docente urbano y las notorias diferencias regionales

\footnotetext{
* Sanchez (1984) menciona que a principios de siglo, en 1903 la formación de escuelas normales tomo primacía; aunque el sistema era muy débil por parte de la acción del Estado quien delegaba la responsabilidad de la formación de docentes en los Hermanos Cristianos. 
de la capacitación de los maestros. Esto en parte se explica por las diferencias en las condiciones de las facultades de educación (cuanto a su calidad y enfoques educativos y a su situación financiera). Parra y Carvajal (1978) plantean la conveniencia de un currículo unificado para la formación de maestros tomando en cuenta la dinámica de la desigualdad existente, tanto a nivel inter-regional como entre lo rural y lo urbano, y por lo tanto no debe promocionarse la existencia de normales de baja calidad en las zonas más deprimidas que es donde deben situarse las de mejor calidad.

Otro aspecto de frecuente análisis se refiere a las deficiencias de la práctica docente como efecto de una inadecuada preparación en las facultades de Educación. Restrepo (1983a; 1983b) señala la necesidad de materializar en el futuro próximo la integración de las facultades de educación dentro de las universidades en un marco pluridisciplinario y se preocupa por la ausencia de investigación e innovación experimental en las facultades, y por lo tanto la poca socialización del estudiantado en estas prácticas. Igualmente recalca la función de la práctica docente como elemento indispensable para el perfeccionamiento de la teoría.

Esta situación del magisterio se agravará aún más a partir de la Reforma Educativa de 1982 (oficializada por decreto 1002 de 1985), ya que según algunos (ver Mockus, ct al , 1985; Quiroz, 1985) la esencia de la reforma es la descalificación del maestro. Por lo tanto se ha venido formando un movimiento de maestros para recuperar el poder que debe tener sobre la educación nacional. Esta reacción en contra de lo que se considera como tecnologías educativas foráneas identifica a los maestros como el único sector que puede ayudar a cambiar lo dispuesto por la Reforma Curricular ya que los alumnos ni los padres son consultados ni tienen la organización para evaluar la reforma. El supuesto básico es que los maestros deben ser agentes de cambio transformadores y propiciadores del mejoramiento de las condiciones de vid de la comunidad. Ver Cristancho de Iriarte et al (1985) para esta posición ampliamente compartida en la literatura.

Los trabajos definitivamente muestran una situación delicada en $\mathrm{k}$ referente a la formación de los maestros y la práctica docente. Aunque parece notarse una actividad en cuanto a búsqueda de cambios se refiere (ver Rojas d Ferro et al, 1985; MEN, 1985) no se sabe con claridad hacia donde se deben dirigir los esfuerzos para superar el problema, y a pesar de los numerosos diagnósticos y reglamentaciones, sigue persistiendo una mala calidad de la educación (Vera y Parra, 1985).

Un trabajo que sirva de complementación a la descripción presentada es el de Gómez y Losada (1984), quienes hacen un riguroso análisis de los conflictos educativos enfatizando el papel que compete a los maestros y su organización, Problemas como cl de los intereses políticos en el nombramiento de los maestros, la grave situación financiera del sistema educativo (en particular el oficial), la remuneración de los maestros, y la politización de las agremiaciones que los representan, han tenido un efecto observable en el deterioro de la calidad del maestro y de la calidad de la educación en general.

Vale la pena señalar que a nivel de las características y condiciones del profesor universitario son prácticamente inexistentes los trabajos. Se encuentran dos, uno de Pelczar (1971) y otro de Vélez y Caro (1986). En el primero se menciona que a principios de la década pasada el desarrollo de la carrera de profesor universitario aún se encontraba en una etapa rudimentaria aunque se observaba un crecimiento en la proporción de profesores de tiempo completo y la notoria disminución del profesor autoritario que enfatiza la memorización de sus conferencias. En el segundo trabajo, 
orientado específicamente hacia el profesorado del cuarto nivel, se destaca que la preparación académica del profesor de postgrado se ha incrementado en los últimos años, y que a pesar de que cerca del 70 por ciento del profesorado de postgrado tienen una dedicación de tiempo completo o exclusiva a esa actividad, la proporción es sustancialmente menor que la observada en México, Venezuela, y principalmente en Brasil.

\section{EDUCACION Y CLASE SOCIAL.}

En el contexto internacional, una de las mayores contribuciones que la sociología ha proporcionado al entendimiento del comportamiento humano se debe a los detallados estudios sobre estratificación social. Vale la pena señalar que este no ha sido el caso en Colombia donde los estudios sobre estratificación social son realmente pocos, y menos aún los que relacionan a la educación con clase social. Sin embargo, es conveniente anotar que en numerosos trabajos se considera la variable educación en su relación con otras características socioeconómicas, con actitudes y comportamientos.

Posiblemente con la misión "Economía y Humanismo" que se llevó a cabo en los años cincuenta es cuando por primera vez se llama la atención sobre la relación entre la educación y la estratificación social; en particular cuando se señala la diferencia existente entre los niveles educativos de los miembros de la clase alta y los de las clases más populares. (Lebret, 1958). Como resultado de esta misión se estimuló el análisis de estudios referentes a la estructura social en el país, y con el apoyo de las políticas de desarrollo, tanto de organismos internacionales como de los gobiernos de la época, en los programas de desarrollo socioeconómico se le da importancia a los efectos de la educación De esta manera expertos como el Profesor Currie (1965)--quien dirigió la primera misión extranjera para planificar el desarrollo del país a fines de la década de los cuarenta - resaltaba la importancia de los efectos de expansión educativa a todos los sectores de la población. En efecto, desde entonces, aunque con diferente énfasis, se ha tomado en cuenta la educación como factor de desarrollo en todos los programas de desarrollo socioeconómico. Se debe anotar, sin embargo, que la inclusión de la educación como instrumento de desarrollo se incluye en dichos planes como producto de aproximaciones ideológicas ligeras y no como resultado de estudios seriamente documentados.

En cuanto a análisis que relacionen la educación con el desarrollo económico, podemos mencionar el de Urrutia (1976), que documenta el papel que la educación jugó en las primeras etapas de la industrialización, argumentando que la educación fue más una causa que un efecto del desarrollo económico en el país.

La supuesta relación entre educación y desarrollo ha servido de base para el diseño de programas educativos. En el contexto rural se han diseñado algunos y quizás el más conocido y difundido sea el de Acción Cultural Popular, ACPO, con el objetivo de motivar al campesino hacia el desarrollo, promoviendo los niveles de conocimiento, participación y productividad. Sin embargo, desde los años setentas, diferentes evaluaciones (ver Morgan et al, 1980; Vélez et al, 1985> han demostrado que la educación aunque es importante para incrementar los conocimientos en lo referente a salud, nutrición, producción, agropecuaria, etc., es un factor muy débil para explicar las puestas en marcha de esos conocimientos, Aspectos como acceso a tierra e ingresos familiares tienen un papel de mayor importancia que la educación. 
En cuanto a los principales hallazgos encontrados en los trabajos sobre educación y clase social se puede comentar que hay una coincidencia con lo descrito en la literatura internacional. De particular importancia se menciona el desigual acceso a la educación dependiendo de la clase social, hasta el punto que la educación puede considerarse como proxy de clase social. Estudios que describen resultados en este sentido se han realizado desde los años setenta y vale la pena destacar los de Rodríguez (1967),-Parra (1969), Rama (1970) y Velásquez (1982).

Cataño (1984) en una buena síntesis sobre el tópico, concretamente sobre educación y diferenciación social, habla de la educación "popular" y la educación "elitista", tanto a nivel universitario como a otros niveles. Igualmente, señala la relación existente entre especialidades que ofrece el sistema educativo y la estratificación social. En efecto, (Psacharopoulos, Vélez y Zabala, 1986) señalan que los bachilleratos técnicos y vocacionales atraen estudiantes de estratos más bajos que los bachilleratos académicos o clásicos de la misma manera el bachillerato diversificado cubre un mayor espectro socioeconómico, incluyendo a estudiantes de estratos bajos.

De otra parte, esta diferenciación también es notoria entre la ciudad y el campo. Concretamente, señala Cataño que la clientela de las escuelas rurales se origina entre los hijos de los aparceros, arrendatarios, minifundistas y jornaleros, de donde se explica la tradicional pobreza de las escuelas rurales. Los hijos de los latifundistas y medianos propietarios se educan en la escuela urbana.

La relación entre educación y movilidad no es tan clara. Históricamente puede detectarse que la naturaleza de la relación ha variado. La expansión educativa observada desde la segunda mitad de este siglo apenas ha tenido una respuesta débil en el mundo del trabajo. En efecto, al inicio de la expansión hubo una correspondencia entre la educación y los ingresos, y los egresados tuvieron la oportunidad de inserción adecuada en el mercado de trabajo. Sin embargo, una vez éste logró niveles de saturación, y al acelerarse la expansión educativa, los egresados tuvieron que presionar hacía abajo, redefiniendo ( $y$ ocupando> ocupaciones que antes exigían una menor credencial educativa. Cataño (1984) argumenta que con la expansión educativa, las desigualdades se trasladan al mercado de trabajo. Y añade que si la educación no es, hoy día, garantía del ascenso social, si puede serlo para retener el status adscrito; y además facilita la movilidad horizontal. Desde la década de los setentas se señalaba que en Colombia (ver García, 1968) el status familiar (educación y ocupación de los padres) parece desempeñar el papel de un filtro seleccionador, indicando que es más fácil que los hijos provenientes de familias con alta posición social desempeñen ocupaciones de bajo prestigio, ya que los provenientes de familias de baja posición ocupen posiciones de alto prestigio.

Reyes y de Gómez (1979) en relación con la educación técnica argumentan que no logra una capacitación para garantizar una movilidad ocupacional; por el contrario parece que se podría generalizar la opinión de que la primera ocupación es bastante definitiva en la historia ocupacional de las personas con este nivel educativo.

Para el caso del egresado universitario, Ocampo (i986) afirma que la mayor oferta producida por la expansión educativa ha podido ser absorbida por el mercado laboral. Sin embargo, es necesario anotar con el autor, que esta absorción (principalmente durante los años setenta) implicó una disminución de los ingresos relativos y una recalificación masiva de los puestos de trabajo en el sector privado, y muy probablemente, un incremento en el desempleo de los bachilleres. En efecto, según un análisis de Kugler y de Gómez (1982) las tasas de desempleo hacia finales de la década pasada ya 
presentaban mayores niveles para las personas con secundaria que para las de primaria e incluso superior. Igualmente se concluye que existen cambios en la composición ocupacional, notándose que hay ocupaciones que fueron desempeñadas por personas con educación primaria y pasaron a serlo por personas con educación secundaria.

En este contexto, se deben mencionar los trabajos de Briones (1980) y Caillods y Briones (1981) que demuestran la poca eficiencia externa de los Institutos Técnicos Industriales en lo referente a satisfacer logros ocupacionales de los egresados. En un estudio más comprensivo (Psacharopoulos, Vélez y Zabalza, (1986) se confirma lo anterior, pero con adición se señala que los INEM tienen aún una menor eficiencia externa. Esta realidad se explica en gran parte por la naturaleza de la demanda que existe en el mercado laboral colombiano.

Gómez (1985), a propósito de la formación profesional, señala que su importancia varía con el relativo desarrollo de los países y en Colombia la introducción de la industria moderna se dio en el contexto de un subdesarrollo educativo, fomentándose así la formación extraescolar para el trabajo. Con la expansión educativa y la alta expectativa de logro educativo que existe en la sociedad colombiana, los egresados de la educación técnica (aunque esta afirmación es válida para todo tipo de educación) muestran interés en continuar estudiando (ver Psacharopoulos, Vélez y Zabalza, 1986) mientras que la industria sigue buscando formación más específica. La relación entre educación y empleo, por lo tanto, no es tan sencilla como se supone por muchos de los planificadores de recursos humanos.

Los anteriores comentarios se refieren más al mundo urbano que al rural. En el campo, quien nace como peón, jornalero, mayordomo, administrador, o propietario, así muere.

Históricamente, cuando se ha observado cambios en esta estructura, la explicación se encuentra en fenómenos que desbordan el papel de la educación (Guerras Civiles en el siglo pasado, la Violencia en este siglo, y más recientemente la producción y comercialización de marihuana y cocaína, etc).

Por esto creemos, como lo anotan varios autores, que la relación educación movilidad no es sencilla y que la educación solo contribuye en parte a la creación de las diferencias de clase.

Urrutia (1974), basado en un análisis de una muestra de 295 casos, argumenta que la educación mejora los ingresos relativos de quien se educa con relación al que no se educa, cuando ambas personas pertenecen a la misma clase social, Por lo tanto concluye que la educación tiene un efecto más claro sobre los ingresos que la posición social de la familia.

Este resultado, creemos, hay que tomarlo con mucha precaución por la manera tan cruda como se operacionaliza el concepto posición social de la familia. Una conclusión más pertinente de ese trabajo debió expresarse de la siguiente manera: la educación de una persona es un mejor predictor de sus ingresos que la educación de sus padres. Al respecto creemos que existen tanto factores sociales como económicos para explicar la relación educación ingresos y es algo que no se debe olvidar. 


\section{CONCLUSIONES}

Con la presente revisión hemos procurado reunir a un conjunto de más de 100 trabajos que consideramos útiles para ilustrar los estudios sobre la relación entre educación y sociedad en nuestro país. El propósito no es otro que el de presentar en unas pocas páginas, aunque hayamos abusado de lo esquemático, los principales hallazgos de los trabajos sobre ese tema.

Debemos anotar que no se han reseñado los trabajos sobre logros cognoscitivos, aspecto que se ha estudiado por parte del ICFES y en particular por algunos proyectos realizados en el Instituto SER de Investigación. Hemos querido dejar este tema para las conclusiones con el fin de destacar la conveniencia del estudio de esta área que mide un producto de la educación muy importante para el desarrollo social. Aunque sea solo a manera de enumeración, queremos presentar algunos de los trabajos más importantes sobre el tema y que apenas abren el camino que se debe continuar en el futuro.

Velandia (1981) quizá presenta el primer estudio sistemático sobre el tema en Colombia, cuando analiza con datos del SNP del ICFES algunos determinantes personales y familiares del rendimiento académico. Amézquita, González y Rojas (1984) también analizan, con la cohorte de bachilleres de 1981, las características socioeconómicas más asociadas con los rendimientos de los resultados en los Exámenes de Estado. Este mismo tema ha sido estudiado en forma recurrente por el ICFES; para un ejemplo ver uno de los informes mas recientes en Guerrero de Molina y Nieto (1987).

Rodríguez (1982) realiza el estudio más comprensivo sobre los logros cognoscitivos en matemáticas y lenguaje entre niños del nivel primario en Colombia.

Rojas (1981) en un estudio realizado en zonas urbanas y rurales se concentra en el papel de la educación formal en el progreso no cognoscitivo del niño concretamente estudia la relación entre la educación y la creatividad, la autoestima, la actitud hacia el maestro y la escuela

Rojas y Vasco (1984) en un estudio de corte Piagetiano analizan la determinación del nivel cognoscitivo de los estudiantes y su relación con el rendimiento en ciencias.

Por último Vélez y Rojas (1987) y Vélez y Rojas (próximo a publicarse) hacen un análisis sobre el logro cognoscitivo de una cohorte de bachilleres tanto en términos de unas pruebas especialmente diseñadas con ese fin como en las pruebas del SNP DEL ICFES, y estudian el impacto que el logro cognoscitivo tiene en el acceso a la educación superior y al mercado laboral.

En general, durante los últimos años son muchos los estudios que sobre el tema se han realizado en Colombia. Sin embargo, es necesario profundizar en casi todos los temas estudiados, especialmente los referentes a la dinámica de la relación educación y sociedad. El vacío más notorio probablemente existe en los temas educación y estratificación social, así como en el de educación y formación de valores. 


\section{BIBLIOGRAFIA}

Aguilar, L.I. y J.J. Perfetti.

"Distribución del Ingreso y Determinantes en el Sector Rural Colombiano" Coyuntura Económica. Vol XVII, N 1, 1987

Alvarado, A. "Les Aspirations Culturelles des Ouvriers de Bogotá" Tesis inédita de Doctorado. Universidad de París. Sorbona ,1973.

Alvarado, A. Educación y Cultura. Ponencia presentada en el 2do. Seminario Nacional de Investigación Educativa, Bogotá, Abril, 1980.

Amézquita,E.,G. Gonzálezy C. Rojas.

Características del Bachiller Colombiano y su Relación con los Exámenes de Estado y su Ingreso a las Instituciones de Educación Superior. Bogotá: Instituto SER de Investigación. 1984.

Benoit, A.

El Bachillerato Colombiano: Aspectos de su Función Ideológica. Bogotá: ICODES, 1968.

Bernal, A. et al.

La Educación en Colombia. Bogota: FERES - CIS, 1965.

Bernal, $\mathrm{H}$.

Teoría y práctica de la Acción Cultural Popular. Bogotá: ACPO. Departamento de Sociología. Documento de Trabajo N² 29. 1976.

Bernal, $\mathrm{H}$.

Educación Fundamental integral Teoría y Aplicación en el Caso de ACPO. Serie EFI N ${ }^{0} 2$. , Bogotá: Editorial Andes, 1978.

Bonilla, E. y E. Vélez.

Mujer y Trabajo Rural en Colombia. Bogotá: Plaza y Janés, 1987.

Bourguinon, $\mathrm{F}$.

"The Role of Education In the Urban Labor Market During Process of Development : The Case of Colombla"Conferencia presentada en el VI Congreso de la Asociación Internacional de Economía . México, Agosto, 1980.

Briones, G.

de los Institutos Técnicos Industriales Bogotá: PNUD -UNESCO- Mineducación, 1980. Caillods, F. y G.Briones. "Educación, Formación Profesional y Empleo: El Sector industrial en Colombia". París: IIPE, 1981.

Cataño, G.

"Desarrollo de la Investigación Sociológica sobre Educación En Colombia". Revista del Centro de Estudios Educativos. Vol. IV, N01, 1974.

Cataño, G.

"Sociología de la educación en Colombia" Revista Colombiana de Educación . N 5, 1980. 
Cataño, G.

"Educación y Diferenciación Social en Colombia". Revista Colombiana de Educación . $\mathrm{N}^{0}$ 14, 1984.

Cristancho de Iriarte, D.P. et al.

Elementos Fundamentales del Perfil teórico del Docente para el Departamento del Huila. Neiva: Universidad Surcolombíana, 1985.

Currie, L.

La Operación Colombiana. Barranquilla: Cámara de Comercio. 2a. edición. 1965.

De Tezanos, A.

Escuela y Comunidad: Un Problema de Sentido. Bogotá, CIUP- CIID. 1983.

DNP-MEN.

Bases para el Desarrollo de un Plan Integrado de Formación y Capacitación de Docentes. Bogotá, 1973.

DNP.

Evolución de Gasto en Educación, 1970-77. UDS-D.E., 002, junio 1979.

Dougherty, Ch.

"El Futuro de la Educación Colombiana: Proyecciones y Prioridades Revista de Planeación y Desarrollo. III,1, 1971.

Eder de Zambrano, D. et al.

Estudio sobre Facultades de Educación. Bogotá. MEN-PNUD, 1982.

Facundo, A.

"Notas para una Metodología y Análisis del Desarrollo de la Investigación Sociológica sobre Educación en Colombia”. En la Sociología en Colombia. Balance y Perspectiva. Bogotá: $\quad$ Asociación Colombiana de Sociología, 1980.

Fals Borda, $\mathrm{O}$

La Educación en Colombia. Bases para su interpretación Sociológica" Monografías Sociológicas $N^{0} 11$. Bogotá: Universidad Nacional -Departamento de Sociología, 1962.

Ferro, M.S.

Mi Vida: Historia de la Vida de una Maestra Rural Colombiana Bogotá: América Latina. 1978.

Flórez, R. y E. Bautista.

El pensamiento pedagógico de los Maestros. Universidad de Antioquia. Comité Central de investigaciones. 1982.

Franco, G.

Rendimiento de las inversiones en Educación en Colombia. Bogotá: U. de los Andes, 1964.

García, C.

Movilidad Ocupacional en Cinco Ciudades Colombianas. Bogotá: Universidad de los Andes, CEDE, Monografía 26. 
Gómez, H. y R. Losada

Organización y Conflicto: La Educación Primaría Oficial enColombia. Ottawa: CIID, 1974.

Gómez, J. et al.

"Autoridad, libertad y aprendizaje: Valores de docentes y discentes en las Educación". Investigación Educativa ". Vol. 1. № 1, 1985.

Gómez, V.M.

Efectos de la innovación Tecnológica sobre el Empleo y la Calificación. Ponencia presentada en el Coloquio Regional sobre la Vinculación de la Educación y el Mundo del Trabajo. Caracas, OREALC, 1985

Guerrero de Molina, D. y Y. Nieto.

"Características del Bachiller y Comportamiento Ante los Exámenes de Estado y el Ingreso a la Educación Post-secundaria". En Memorias 11 Encuentro de Investigadores Sobre Calidad de la Educación Media. Bogotd: FES-CIID, 1987.

Haller, T.

Education and Rural Development ín Colombia.Tesis Inédita de Doctorado. Lafayette: Purdue University, 1972.

Horn, R.

'The Earning Impact of Education Experience and Formal Job Training SENA) Among the Self-Employed in Colombia". Washington Education and Training Department. The World Bank, 1986.

Inkeles, A. y D.H. Smlth.

Becoming Modern. Cambridge. Harvard University Press, 1974.

Jiménez, E. y B. Kugler.

Evaluación Económica de un Sistema Nacional de Formación Profesional. Servicio Nacional de Aprendizaje de Colombia (SENA).Washington: The World Bank. Discussion Paper. Education and Training Series, 1986.

Kugler, B.

"Una revisión crítica de investigaciones hechas en Colombia sobre Educación y Economía". Revista Colombiana de Educación. Nª 5, 1 semestre 1980.

Kugler, B.

'The Influence of Education on Labor Incomes: The Colombian Case". En Economic Policy and Income Distribution in Colombia. RA. Berry y R.

Soligo (eds) .Boulder: Westview, Press, 1980.

Kugler, B. y MI. de Gómez.

"La Educación Secundarla en el Mercado de Trabajo". Revista Colombiana de Educación $\mathrm{N}^{0} 10,2$ semestre, 1982.

Lebret, L.J.

Estudio sobre las condiciones del Desarrollo de Colombia. Bogotd: Editorial Cromos, 2 Vols. 1958.

Losada, R.

"Los maestros oficiales de Colombia frente al mundo Político ". Revista Colombiana de Educación $\mathrm{N}^{0} 11,1983$. 
Maldonado, 1-1.

"La Rentabilidad de los Cursós de Aprendizaje del SENA" Tesis inédita. Bogotá: Universidad Nacional, 1973.

Manheim, K.

Ensayos de Sociología de la Cultura.' Madrid: Ediciones Aguilar, 1957.

Martínez, A.

El Maestro y la Instrucción Pública en el Nuevo Reino de Granada, 1767-1809. Bogotá: CIUP, 1981.

Martínez, A.

"La Escuela, Los procesos de Enseñanza y la Alternativa del Movimiento Pedagógico". En Educación, Pedagogía y Cultura. Bogotá: Escuela Nacional Sindical, 1984.

MEN

"Qué es el Modelo Pedagógico Integrado para las Normales Demostrativas?" Boletín Informativo MEN, 1985.

Mockus, A. et al.

“La Reforma Curricular y el Magisterio" Educación y Cultura. Vol. 1 N4, 1985.

Mohan, R.

Work. Wages and Welfare ín a Developing Metropolis New York: Oxford University Press, 1987.

Morgan, R. et al

Evaluación de Sistemas de Comunicación Educativa. Serie EFI. N $N^{0} 5$ Bogotá: Editorial Andes, 1980.

Muñoz, J.A.

“La des-socialización del Niño". Revista Colombiana de Educación. N0 12, 1983.

Muñoz, JA.

La recuperación de la Cultura: Un Compromiso de la Escuela. Bogotá. UP’N. 1984.

Nieto, D.

La Educación en el Nuevo Reino de Granada. Bogotá: Editorial Santa Fé, 1955.

Ocampo, J.

Educación, Humanismo y Ciencia. Tunja: Ediciones La Rana y el Aguila, 1978.

Ocampo, JA.

"Empleo, Desempleo e Ingresos de la Fuerza de Trabajo Universitaria, 1976 - 1985". En Crisis en la Educación Superior (Memorias de la reunión ICED/ IASED celebrada en Bogotá), abril de 1986.

OSPE-MEN

Presupuesto Definitivo para Educación por Grandes Programas, 1970 -1980. Septiembre 1980. 
Parra, R.

"Clases Sociales y Educación en el Desarrollo de Colombia". UN .Revista de la Dirección de Divulgación Cultural. N³, 1969.

Parra, R

La profesión del maestro y el desarrollo nacional. Bogotá Ministerio de Educación Nacional. UNESCO-PNUD. Mimeo 1979.

Parra, R.

"La Imagen soda! del maestro". Educación y Cultura. CEID, N 3, 1985.

Parra, R. y M. E Carvajal.

Bases Sociales para la Formación a Distancia de los Maestros

Colombianos. Bogotá: Informe presentado al MEN- OSPE, 1978.

Parra, R. y L. Zubíeta.

La Imagen del Maestro en la Escuela CampesIna.Bogotá: CIUP. 1981

Parra, R. et al

Las Relaciones Sociales en la Práctica Pedagógica de las Escuelas Normales. Bogotá, CIUP, 1981.

Pasa, D.

El Maestro Rural. Bogotá: Fundación Friedrich Naurnann, 1979.

Pelczar, R.

"The Uníversity Professor in Latin América: A New Look at an Oid Stereotype". Trabajo presentado en la reunión de la Comparative and International Education Society en Chicago, Febrero, 1971.

Porras Troconís, G.

Historia de la Cultura en el Nuevo Reino de Granada Sevilla: Escuela de Estudios Hispano Americanos de Sevilla. 1952.

Psacharopoulos, G.

Returns to Educatíon. An International Comparison. Amsterdam: Elsevier, 1973.

Psadiaropoulos, G

Returns te educatlon: An Update Interna tíonalComparison" Comparative Education. Vol 17. $\mathrm{N}^{0} 3,1981$

Psacharopoulos, G

"Education and Prívate Versus Public Sector Pay". Labor and Society Vol. 8, N 2 Abril junio, 1983.

Psacharopoulos, G., E. Vélez y A. Zabalza.

Una Evaluación de la Educación Media Diversificada en Colombia. Bogotá: Rojas Eberhard Editores, 1986.

Psacharopoulos, J., A.M. Arrlagada y E. Vélez. 
"Eamníngs and Education Among the self- Employed ín Colombia" Trabajo presentado en la Reunión Anual de la Eastern Economic Association Washington, Marzo, 1987.

Puryear, j.

"Vocational Trainíng and Eamnings in Colombia: doesa SENA Effect Exist?" Comparative Educalion Review. vol. 23, N² 1979.

Quiroz, A.

"Una pérdida de Identidad Cultural del Maestro: Decreto 1002". Educación y Cultura. $\mathrm{N}^{0} 4$, 1985.

Rama, G. W.

El Sistema Universitario en Colombia Bogotá: Universidad Nacional de Colombia, 1970.

Restrepo, 8.

"Facultades de Educación : Situación Actual y Tendencias Hacia el Futuro". Revista de Estudios Educativos. N018 1983 a.

Restrepo, 8.

"Práctica como Reflexión y Práctica como Investigación Acción: Una Propuesta de Práctica Profesional para la Formación de Docentes a Distancia”. Educación Superior y Desarrollo. Vol. 1, 1983 b.

Reyes, A. y M.I.de Gómez.

Roles y Posibilidades de la Educación técnica: El Caso de Colombia. Bogotá: CCRP, 1979.

Rodríguez, J.

Religión y Cambio Social en el Bachillerato Colombiano Monografías Sociológicas N ${ }^{0} 25$. Bogotá: Universidad Nacional. Departamento de Sociología, 1967.

Rodríguez, J.

Educación Católica y Secularización en Colombía.Bogotá: Editorial Stella, 1970.

Rodríguez Valderrama, J.

El Logro en Matemáticas y Lenguaje en la Educación Primaria en Colombia. Bogotá: Instituto SER de Investigación, 1982.

Vélez, E. y C. Rojas.

El Bachillerato Colombiano: Que Saben. Qué Desean y Qué Logran sus Estudiantes. Bogotá: Próximo a Publlcarse.

Vera, CA. y F. Parra.

"Contrlbuclón a un Análisis de la Capacitación docente y la Práctica Pedagógica". Invastigación Educativa. Vol. 1, N01. 1985.

Williamson, R.

"El Estudiante Colombiano y sus Actitudes". Monografías Soclóloglcas $\mathrm{N}^{0} 13$. Bogotá: Universidad Nacional . Departamento de Sociología, 1962.

Zuluaga, O.L 
El Maestro y el Saber Pedagógico en Colombia (1821-1948). Bogotá, COLCIENCIAS, Informe de Investigación, 1982. 